\title{
La libre circulación de resoluciones en la UE en el caso de la sustracción internacional de menores
}

\section{The free circulation of resolutions in the $E U$ in the case of abduction international minors}

\author{
María GonZÁLEZ MarimóN ${ }^{1}$
}

\section{RESUMEN}

El presente artículo aborda la regulación actual y futura del sector de reconocimiento y ejecución de resoluciones extranjeras en materia de sustracción internacional de menores en el ámbito de la Unión Europea. Materia específica que motivó la previsión de un régimen de eficacia extraterritorial de resoluciones judiciales distinguido $-\mathrm{y}$ privilegiado- al del régimen genérico de las resoluciones en materia de responsabilidad parental. Recientemente, el instrumento aplicable en la materia, el Reglamento 2201/2003 (Reglamento Bruselas II bis) ha sufrido un importante proceso de reforma que ha culminado con la aprobación de un nuevo Reglamento, el Reglamento 2019/1111. En este trabajo abordaremos el complejo entramado de fuentes en la materia, con la interacción tanto de fuentes de la UE como de convenios internacionales, principalmente del Convenio de La Haya de 1980, analizando tanto el régimen actual como las modificaciones apuntadas. Con todo ello, se irán apuntando algunas de las tendencias actuales y futuras del tercer sector del Derecho Internacional privado en el proceso de construcción del espacio de libertad, seguridad y justicia de la UE.

Palabras clave: Reconocimiento y ejecución de resoluciones extranjeras, Unión europea, Reglamento Bruselas II bis y ter, sustracción internacional de menores.

\section{ABSTRACT}

This article faces the present and future regulation of the recognition and enforcement of judicial resolutions in the matter of international child abduction in the European Union. Specific subject which motivated a singular -and privileged- regime of recognition and enforcement, different from the general regime in the matter of parental responsibility. Recently, the applicable instrument, the 2201/2003 Regulation (Brussels IIa Regulation), has suffered an important recast which has ended with the promulgation of a new Regulation, the 2019/1111 Regulation. Therefore, in this work we will deal with the complex interaction between the legal sources, both of EU sources and international conventions, notably the 1980 Hague Convention, analysing not only the present regimen but also the abovementioned modifications. This analysis will allow us to conclude with some of the actual and future tendencies of the Private International Law third sector in the process of construction of a free area of freedom, security and justice in the EU.

Keywords: Recognition and enforcement of judicial resolutions, European Union, Brussels II a Regulation, Brussels II b Regulation, international child abduction.

\footnotetext{
Personal Investigador en Formación (FPU), Ayuda para la Formación del Personal Universitario (FPU), financiada por el Ministerio de Ciencia, Innovación y Universidades de Espańa. Departamento de Derecho Internacional “Adolfo Miaja de la Muela”, Área de Derecho Internacional privado, Universitat de València. Correo electronico: maria.gonzalez-marimon@uv.e
} 


\section{Introducción: Un espacio europeo de seguridad y justicia en construcción}

El presente artículo aborda la regulación actual y futura del sector del reconocimiento y ejecución de resoluciones extranjeras en materia de sustracción internacional de menores en el ámbito de la Unión Europea (en adelante, UE). Materia específica que se ubica dentro de la genérica "responsabilidad parental" del menor y que, precisamente por su especialidad, motivó la previsión de un régimen de eficacia extraterritorial de resoluciones judiciales distinguido $-\mathrm{y}$ privilegiado- al del régimen genérico de las resoluciones en materia de responsabilidad parental. Sin embargo, este régimen ha suscitado una importante problemática que lleva a reflexionar directamente sobre los principios base del sistema de reconocimiento y ejecución de resoluciones judiciales de la UE. Tal es así que, recientemente, el instrumento aplicable en la materia, el Reglamento No 2201/2003 del Consejo, de 27 de noviembre de 2003, relativo a la competencia, el reconocimiento y la ejecución de resoluciones judiciales en materia matrimonial y de responsabilidad parental ${ }^{2}$ (en adelante, Reglamento Bruselas II bis) ha sufrido un importante proceso de reforma que ha culminado con la aprobación de un nuevo Reglamento ${ }^{3}$. En este trabajo abordaremos las claves del nuevo sistema. Este estudio nos permitirá, adicionalmente, ir apuntando algunas de las tendencias actuales y futuras del tercer sector del Derecho Internacional privado (en adelante, DIPr) en el ámbito de la UE.

\subsection{Algunas precisiones conceptuales previas}

Antes de comenzar el análisis anunciado resulta pertinente realizar tres precisiones conceptuales en relación tanto de la materia tratada como del ámbito de aplicación de la misma en la UE.

En primer lugar, conviene recordar que la sustracción internacional de menores se produce cuando un progenitor decide trasladar o retener ilícitamente a un menor en un Estado distinto al de su residencia habitual. Lo anterior, contraviniendo los derechos de custodia, establecidos de conformidad con la legislación de la residencia habitual del menor.

Este fenómeno continúa siendo un problema en nuestras sociedades, viéndose incrementado su número y trascendencia, en el caso de Europa, debido a la mayor facilidad de movimiento de personas, especialmente en el territorio de la UE. En concreto, en este trabajo estamos abordando aquellas situaciones en las que un progenitor traslada o retiene ilícitamente a un menor en un Estado miembro de la UE distinto al del Estado miembro de la UE de residencia habitual del menor. En estas situaciones, desde la óptica del DIPr, se activarán una serie de instrumentos internacionales que pretenden resolver la situación, generalmente mediante el retorno inmediato del menor al lugar de su residencia habitual.

En segundo lugar, precisamente en el marco de este entramado de normas es donde se ubica, con carácter preferente, la aplicación de la normativa de la UE, el ya mencionado Reglamento Bruselas II bis. Efectivamente, el proyecto europeo ha llegado a un nivel de integración tal que permite a las instituciones de la UE regular este sector normativo. Con la entrada en vigor del Tratado de Ámsterdam ${ }^{4}$ y posteriormente del Tratado de Lisboa ${ }^{5}$, el Derecho Primario de la UE recoge, dentro del proceso de

DO L 338 de 23 de diciembre de 2003, cde DO L 82, de 22 de marzo de 2013.

3 Reglamento (UE) 2019/1111 del Consejo, de 25 de junio de 2019, de 25 de junio de 2019, relativo a la competencia, el reconocimiento y la ejecución de resoluciones en materia matrimonial y de responsabilidad parental, y sobre la sustracción internacional de menores (versión refundida), DO L 178/1 de 2 de julio de 2019.

4 Tratado de Ámsterdam por el que se modifican el Tratado de la Unión Europea, los Tratados Constitutivos de las Comunidades Europeas y determinados actos conexos, DO C 340, de 10 de noviembre de 1997.

5 Tratado por el que se modifican el Tratado de la Unión Europea y el Tratado Constitutivo de la Comunidad Europea, DO C 306, 17 de diciembre de 2007. 
consolidación de un espacio judicial europeo -espacio de libertad, seguridad y justicia-, la posibilidad expresa para legislar en materia de DIPr desde la UE. Actualmente, la base jurídica que permite al legislador de la UE adoptar instrumentos en materia de DIPr se encuentra en el Título V (artículos 67.4 y 81) del Tratado de Funcionamiento de la Unión Europea ${ }^{6}$ (en adelante, TFUE). El precepto diseńa un conjunto de medidas diversas a desarrollar en el ámbito de la cooperación judicial en materia civil con repercusión transfronteriza ${ }^{7}$.

Por último, es imprescindible hacer alusión a los principios propios de la cooperación judicial en materia civil cuando entramos en materia de reconocimiento y ejecución de resoluciones judiciales entre Estados miembros de la UE. Desde la perspectiva del DIPr, uno de los objetivos fundamentales del Derecho de la UE es la libre circulación de resoluciones judiciales. Un objetivo esencial dentro del proceso de consolidación de este espacio jurídico integrado que ha sido elevado por su trascendencia a la condición de "quinta libertad comunitaria” (Iglesias y Desantes, 1986, p. X), junto a la libre circulación de mercancías, trabajadores, servicios y capitales sobre los que se asienta la Unión. Esta quinta libertad comunitaria queda sustentada por los principios de reconocimiento mutuo y confianza judicial entre las Administraciones de Justicia de los Estados miembros en el denominado "espacio de libertad, seguridad y justicia de la UE" 8

Su logro efectivo exige la simplificación de los trámites necesarios para reconocer las resoluciones judiciales dictadas en el espacio de la UE, de un modo rápido y automático, estableciendo para ello un conjunto de normas comunes en los distintos instrumentos comunitarios diseńados sobre una amplia pluralidad de cuestiones. En todas ellas se ha buscado asegurar el reconocimiento automático de las resoluciones judiciales dictadas por los tribunales de los distintos Estados de la Unión y su posterior ejecución. El proceso, sin embargo, ha sido largo y complejo, y a fecha de hoy está todavía inconcluso. De hecho, nos encontramos en una encrucijada.

\subsection{Las claves del modelo}

A primera vista, y de manera global, puede apuntarse que la automaticidad del reconocimiento ha sido proclamada sin especial problematismo en los distintos instrumentos de la UE en la materia. Su práctica tampoco ha planteado un elevado nivel de tensiones?.

No puede decirse lo mismo, sin embargo, del ámbito de la ejecución de las resoluciones judiciales, donde el proceso de integración no es ni ha resultado tan sencillo. El objetivo final del proceso de armonización del DIPr europeo debería conducir paulatinamente a la supresión del exequátur o declaración de ejecutividad entre los países miembros de la UE, asegurando la ejecución automática en un determinado Estado miembro de las resoluciones judiciales dictadas por los órganos jurisdiccionales de otro Estado miembro de la UE. Esto, sin embargo, no puede decirse que haya sido alcanzado todavía de forma plena, ni que vaya a serlo en un futuro inmediato ${ }^{1011}$.

\footnotetext{
Versión Consolidada del Tratado de Funcionamiento de la Unión Europea, DO C 326/47, de 26 de octubre de 2012.

Véase Arenas (2019), Campuzano (2013), González (2004), Iglesias (1998) y Sánchez (2012 y 2019).

Véase Álvarez (2018), De Hoyos (2007), Iglesias (2010), Michinel (2013), Ordóñez (2014), Gómez (2006) y Pertegás (2019).

Véase Gayo (2013).

10 Entre otras razones, la histórica consideración del ejercicio de la potestad jurisdiccional como una manifestación de la soberanía del Estado se encuentra en la base de las reticencias a avanzar en la supresión efectiva del exequatur en el interior de la UE. A ello, además, habría que sumarle una segunda dimensión, ciertamente compleja, como son las divergencias en las legislaciones de los Estados miembros, tanto en cuanto al fondo de los derechos regulados como en el propio proceso de ejecución (Espinosa, 2007, p. 215).

11 Véase Caamiña (2011), Font (2004) y Garau (2004).
} 
En el marco de este proceso de conformación del espacio europeo de libertad, seguridad y justicia, el Reglamento Bruselas II bis presenta unas peculiaridades singulares tanto por la materia que aborda, directamente vinculada a la esfera más privada de las personas, como por las características de las soluciones diseñadas en él. Este instrumento de fuente institucional contiene unas normas unificadas de competencia judicial internacional y reconocimiento y ejecución de resoluciones extranjeras en materia matrimonial y de responsabilidad parental en el que se diseñan. Además de ciertas normas sobre la sustracción internacional de menores que han generado un elevado nivel de complejidad en su puesta en práctica. Esto no solo por sus peculiaridades, sino también, y de forma relevante, por dos motivos ajenos a la propia realidad normativa de la Unión.

En primer lugar, y en relación con las soluciones incluidas en el texto reglamentario en materia de sustracción internacional de menores -junto con los derechos de visita-, en la que un reglamento de la UE recogió por primera vez la supresión del exequátur ${ }^{12}$. Con ello el legislador de la UE tomó una peculiar decisión: mantener dos regímenes diferenciados de ejecución en un mismo reglamento: uno genérico en materia de responsabilidad parental -manteniendo el exequátur- y otro privilegiado para las materias concretas mencionadas.

Junto a ello, las normas del Reglamento interactúan con otros textos normativos de carácter convencional que, emanados de otros centros codificadores distintos a la UE vinculan, sin embargo, a los distintos Estados miembros de la Unión. En el ámbito de la Conferencia de La Haya de DIPr, son de necesaria mención tanto el Convenio de La Haya de 25 de octubre de 1980 sobre aspectos civiles del secuestro internacional de menores ${ }^{13}$ (en adelante, Convenio de La Haya de 1980) como el Convenio de La Haya de 19 de octubre de 1996 relativo a la competencia, la ley aplicable, el reconocimiento y la ejecución y la cooperación en materia de responsabilidad parental y medidas de protección de los niños (en adelante, Convenio de La Haya de 1996) ${ }^{14}$.En segundo, y no menos trascendente, porque la realidad regulada por este conjunto de disposiciones es objeto de interpretación por tres niveles judiciales diversos, los tribunales nacionales, el Tribunal de Justicia de la UE y el Tribunal Europeo de Derechos Humanos ${ }^{15}$. La interacción de estos dos, precisamente, será de especial relevancia en esta materia ${ }^{16}$.

\section{El complejo entramado de fuentes en materia de sustracción ilícita internacional}

La compleja confluencia de fuentes de origen y ámbito diferenciados hace necesario un primer estudio de las mismas, para poder comprender el alcance de la problemática surgida en la ejecución de resoluciones judiciales en relación con la sustracción internacional de menores en el ámbito del Reglamento Bruselas

12 Posteriormente, otros Reglamentos han incorporado la eliminación del exequátur, como por ejemplo el Reglamento (CE) No 805/2004 del Parlamento Europeo y del Consejo, de 21 de abril de 2004, por el que se establece un título ejecutivo europeo para créditos no impugnados. BOE No 202, de 24 de agosto de 1987, cde BOE No 155, de 30 de junio de 1989, y BOE No 21, de 24 de enero de 1996.

BOE de 2 de diciembre de 2010.

15 El TJUE se encarga de la correcta aplicación e interpretación del Derecho de la UE, sobre todo mediante la resolución de cuestiones prejudiciales planteadas por órganos jurisdiccionales de los Estados miembros de la UE. EL TEDH es el garante de los derechos humanos contenidos en el Convenio Europeo de Derechos Humanos (CEDH) del Consejo de Europa -organización internacional que promueve los derechos humanos en el continente europeo- y conoce de demandas individuales para controlar ex post la presunta violación de un derecho contenido en el CEDH.

16 Para un análisis sobre la interrelación de los dos sistemas de protección de derechos fundamentales a nivel europeo véase López (2017). Con carácter específico en materia de sustracción internacional de menores, véase Bouza (2012), González Marimón (2019b), Herranz (2012), Hess (2014-2015) y Porcheron (2015). 
II bis. Precisamente por el objeto de estudio nos centraremos en las principales fuentes relacionadas con la materia: el Convenio de La Haya de 1980 y el Reglamento Bruselas II bis ${ }^{17}$.

\subsection{El Convenio de la Haya de 1980: instrumento de referencia en materia de sustracción internacional de menores}

El Convenio de La Haya de 1980 es, sin duda, el instrumento normativo de referencia en materia de sustracción internacional de menores, tanto en Europa como fuera de ella ${ }^{18}$. Su éxito reside en el diseño de un mecanismo de retorno del menor, basado en la cooperación entre Estados contratantes, cuya finalidad última es el retorno del menor en el menor plazo posible ${ }^{19}$. En consecuencia, el Convenio no contiene normas relativas a los sectores clásicos de DIPr y particularmente, en lo que interesa al objeto de este trabajo, de reconocimiento y ejecución de resoluciones extranjeras.

Como decíamos, su sistema apuesta por una acción directa de retorno del menor, de tal manera que la regla general es la obligación del Estado contratante en el que se encuentra ilícitamente el menor, de ordenar su retorno inmediato al lugar de su residencia habitual ${ }^{20}$. El sistema se completa con una serie de excepciones al retorno del menor en base a circunstancias excepcionales ${ }^{21}$. Efectivamente, aunque el Convenio prioriza con su regla general el retorno del menor, como manifestación de su interés superior, flexibiliza su respuesta, permitiendo el no retorno del menor en los mencionados supuestos excepcionales (McEleavy, 2005, p. 34).

Especial importancia en la práctica ha suscitado la excepción prevista en el artículo 13 b del Convenio, el cual permite denegar el retorno del menor en caso de que tal retorno exponga al menor a un grave riesgo o a un peligro físico o psíquico o que de cualquier otra manera le coloque en una situación intolerable. En la práctica se han detectado ciertos problemas de interpretación en relación con esta excepción al retorno, relacionados con en un presunto abuso de la excepción por grave riesgo del menor por ciertas autoridades nacionales ${ }^{22}$.

\subsection{El Reglamento Bruselas II bis: complementariedad (en la UE) con el Convenio de la Haya de 1980 en materia de sustracción internacional de menores}

Ya hemos señalado que el Reglamento Bruselas II bis se aplicará en el supuesto de que se produzca un traslado o retención ilícito en un Estado miembro de la UE de un menor que tenía su residencia habitual en otro Estado miembro de la UE. Instrumento que, dentro de sus normas de competencia judicial internacional y reconocimiento y ejecución de resoluciones extranjeras en materia de responsabilidad parental, incorpora ciertas normas específicas relativas a la sustracción ilícita internacional. Para ello, el

\footnotetext{
17 El Convenio de La Haya de 1996 recoge los tres sectores de DIPr en materia de responsabilidad parental. Desde el punto de vista de las sustracciones internacionales de menores producidas en el ámbito territorial de la UE -es decir, traslado ilícito de un menor de un Estado miembro de la UE a otro-, el Convenio de La Haya de 1996 juega un papel importante en cuanto a la determinación del Derecho aplicable. Sin embargo, desde el punto de vista del reconocimiento y ejecución de resoluciones extranjeras no tiene virtualidad práctica, puesto que las normas del Reglamento Bruselas II bis, en la materia desplazan a las del Convenio.

18 Con 101 Estados contratantes en 2019.

19 Para un estudio de este Convenio, véase Beaumont (1999), Lapiedra (2008) y Pérez (1982).

20 Véase artículo 12 párrafo $1^{\circ}$ del Convenio de La Haya de 1980.

21 Las excepciones al retorno están previstas en los artículos 12, 13 y 20 del Convenio de La Haya de 1980.

22 Los problemas de aplicación práctica de la excepción de retorno del artículo 13 b) del Convenio de La Haya de 1980 han sido puestos de relieve por numerosa doctrina. En este sentido, véase Forcada (2016 a y b) y Guzmán (2014-2015). Hay autores que afirman que el problema no es tanto el propio artículo $13 \mathrm{~b}$ ), sino la deficiente efectividad de las medidas de protección, tanto para el menor como de su progenitor (en casos, por ejemplo, de violencia doméstica o de género). En este sentido, véanse Reig (2018) y Trimmings (2013).
} 
legislador de la UE se basa en el régimen ya existente del citado Convenio de La Haya de 1980, es decir, el Reglamento Bruselas II bis remite al Convenio, pero a su vez introduciendo ciertas modificaciones ${ }^{23 .}$

Las modificaciones introducidas por el Reglamento respecto al régimen convencional son esencialmente de carácter procesal. El legislador de la UE ańade una serie de garantías en la articulación procesal del mecanismo de restitución previsto en el Convenio de La Haya de 1980. De tal manera, para su correcta interpretación deben aplicarse ambos instrumentos conjuntamente24.

Estas alteraciones están ubicadas en el artículo 11 del Reglamento, el cual se ocupa del proceso de restitución de un menor, tras un traslado ilícito producido dentro del ámbito espacial de la UE. Siguiendo el espíritu del Convenio, la restitución deberá solicitarse a las autoridades competentes del Estado miembro donde ha sido trasladado ilícitamente o retenido. Ahora bien, como hemos anticipado, se introducen ciertos cambios que, fundamentalmente, tienen dos consecuencias. En primer lugar, se refuerza en mayor medida el retorno del menor y, en segundo lugar, se refuerza la prioridad de las decisiones tomadas por el Estado miembro de la UE de residencia habitual del menor, con carácter anterior al traslado o retención ilícito (Magnus y Mankowski, 2012, p.129).

Concretamente, los 5 primeros apartados del artículo 11 del Reglamento contienen las siguientes cuatro modificaciones:

1) Se refuerza el derecho de audiencia del menor, siempre que sea conveniente en función de su edad y madurez.

2) Se refuerza el derecho de audiencia de la parte requirente, que normalmente será la parte que solicita la restitución del menor.

3) Se declara el carácter urgente del procedimiento, con un plazo máximo de 6 semanas para su resolución.

4) Se limita la excepción al no retorno prevista en el artículo 13 b) del Convenio de La Haya de 1980 -grave riesgo para el menor-, de tal manera que los órganos jurisdiccionales no podrán negar la restitución de un menor basándose en este precepto si se demuestra que se han adoptado "medidas adecuadas para garantizar la protección del menor tras su restitución”.

La originalidad de la remisión de una fuente institucional a una fuente convencional no fue la única novedad introducida por el Reglamento Bruselas II bis. Efectivamente, el legislador europeo optó por un delicado reparto competencial en materia de sustracción internacional de menores, intentando fortalecer a los órganos jurisdiccionales del Estado de residencia habitual del menor, con carácter previo al traslado o retención ilícita (Reig, 2008, p. 231). Este peculiar reparto competencial se llevó a cabo mediante la introducción de un complejo procedimiento en los apartados 6 a 8 del artículo 11 del Reglamento, el llamado mecanismo de prevalencia o de última palabra. Pensando en aquellos casos en los que se dicte la no restitución del menor en base al artículo 13 del Convenio de La Haya de 1980, se da la posibilidad al órgano jurisdiccional competente sobre el fondo -que normalmente será el Estado de residencia habitual

23 Con ello, el legislador de la UE modificó su postura respecto al Reglamento (CE) No 1347/2000 del Consejo, de 29 de mayo de 2000, relativo a la competencia, el reconocimiento y la ejecución de resoluciones judiciales en materia matrimonial y de responsabilidad parental sobre los hijos comunes, derogado por el actual Reglamento Bruselas II bis (DO L 160 de 30 de junio del 2000). El artículo 4 remitía directamente al Convenio de La Haya de 1980 para los supuestos de sustracción ilícita internacional.

24 Tal y como seńala la Comisión Europea: "cuando un órgano jurisdiccional de un Estado miembro reciba una solicitud de restitución de conformidad con el Convenio de La Haya de 1980, aplicará las normas del mismo tal y como queda completado por los apartados 1 a 5 del Reglamento". (Comisión Europea, p. 53)3. 
del menor previamente al traslado ilícito- para que resuelva definitivamente y con prioridad sobre el retorno del menor.

La prioridad se consigue precisamente mediante la eliminación del exequátur del mecanismo de prevalencia del artículo 11.8 del Reglamento Bruselas II bis. Es decir, de la resolución ordenando el retorno dictada por el órgano competente (que generalmente será el del Estado de residencia habitual del menor previa al traslado o retención ilícita), con carácter posterior a una resolución de no retorno, basada en un motivo de excepción al retorno del Convenio de La Haya de 1980 y emitida por el Estado en el que se encuentra ilícitamente el menor.

Podemos anticipar que el llamado mecanismo de prevalencia respondía a dos finalidades esenciales. En primer lugar, la superación de los excesos en la aplicación de las excepciones al retorno del menor del Convenio de La Haya de 1980, todo ello bajo el prisma de la consecución del interés superior del menor, mediante el retorno inmediato a su lugar de residencia habitual. En segundo lugar, responde al objetivo de la libre circulación de resoluciones judiciales dentro del espacio de libertad, seguridad y justicia de la UE. Efectivamente, se combinaron los criterios del interés del menor en el retorno inmediato y el principio de reconocimiento mutuo como justificación de la ejecución directa de un sistema cuasi automático de retorno del menor.

Ciertos sectores de la doctrina, desde un inicio, plantearon sus reticencias respecto al sistema diseñado por el legislador europeo ${ }^{25}$. En la actualidad, tras trece ańos de aplicación del Reglamento Bruselas II bis y su respuesta a la sustracción internacional de menores, también la jurisprudencia ha evidenciado varios problemas en su aplicación práctica. La eliminación del exequátur y la consecuente aplicación cuasi automática para las órdenes de retorno del menor pueden no garantizar la protección de los derechos fundamentales del menor y la consideración de su interés superior en todos los casos.

Siendo consciente el legislador europeo de los mencionados problemas de aplicación del Reglamento Bruselas II bis, el 30 de junio de 2016 la Comisión Europea presentó su Propuesta de Refundición del Reglamento (CE) No 2201/2003 del Consejo, de 27 de noviembre de 2003, relativo a la competencia, el reconocimiento y la ejecución de resoluciones en materia matrimonial y de responsabilidad parental, por el que se deroga el Reglamento (CE) No 1347/2000 (en lo sucesivo, Propuesta de Refundición del Reglamento Bruselas II bis ${ }^{26}$. Este proceso de reforma culminó en junio de 2019 con la aprobación del Reglamento (UE) 2019/1111 del Consejo, de 25 de junio de 2019, relativo a la competencia, el reconocimiento y la ejecución de resoluciones en materia matrimonial y de responsabilidad parental, y sobre la sustracción internacional de menores (versión refundida), en adelante, Reglamento Bruselas II ter ${ }^{27}$.

\section{Reconocimiento y ejecución de resoluciones judiciales en el RBII bis}

Tal y como se ha señalado en el apartado anterior, el legislador europeo tan solo optó por la eliminación del exequátur para ciertas resoluciones muy concretas del Reglamento Bruselas II bis. De ello se extrae que, en materia de responsabilidad parental, el Reglamento Bruselas II bis tiene la peculiaridad de

25 Entre otros se puede citar a Caro (2011) y López de Tejada (2011).

26 Véase Propuesta de Reglamento del Consejo relativo a la competencia, el reconocimiento y la ejecución de resoluciones en materia matrimonial y de responsabilidad parental, y sobre la sustracción internacional de menores (refundición) [COM (2016) 411 final, 2016/0190 (CNS)].

$27 \quad$ DO L $178 / 1$ de 2 de julio de 2019. 
combinar dos modalidades de reconocimiento y ejecución de resoluciones extranjeras. Efectivamente, el capítulo III distingue entre el régimen de reconocimiento y ejecución de las resoluciones en materia de responsabilidad parental (régimen general), y la ejecución para las resoluciones en materia de derechos de visita y sustracción internacional de menores (régimen especial) (Esplugues et al., 2020, p. 437 y ss).

El modelo diseñado en el Reglamento Bruselas II bis presenta unas peculiaridades propias, motivadas por la presencia de este régimen doble. Esta realidad es peculiar de este Reglamento y lo diferencia del resto de instrumentos europeos en materia de reconocimiento y ejecución de resoluciones judiciales. Del régimen diseñado por el Reglamento, además debemos añadir dos datos introductorios que manifiestan la peculiaridad de la materia.

En primer lugar, la justificación para la eliminación de la declaración de ejecutividad no solo reside en el objetivo de la libre circulación de resoluciones judiciales y los principios mencionados -reconocimiento mutuo y confianza judicial-, sino que la finalidad última de esta medida sería el principio del interés superior del menor (Ancel y Muir, 2005, p. 569 y ss.). Efectivamente, la eliminación del exequátur supone una reducción de los tiempos procesales y ello beneficia directamente a los intereses del menor, puesto que, en las materias concretas de derechos de visita y sustracción internacional de menores, el paso de tiempo es muy perjudicial para el menor.

En segundo lugar, es esencial tener presente que el legislador de la UE no solo optó por la supresión del exequátur, sino que, dentro de las diferentes opciones que podría haber considerado, optó por una medida de integración muy elevada: la eliminación del exequátur "en términos absolutos", esto es, sin la posibilidad de alegar ningún motivo de oposición en sede de ejecución. Quizá ha sido este punto el que mayores problemas ha generado en la práctica, como se verá en este artículo.

Precisamente por lo expuesto hasta el momento, la sustracción internacional de menores o, mejor dicho, su tratamiento por el legislador de la UE, merece un análisis exhaustivo. Y es que, a través del estudio del régimen, no tanto de reconocimiento, sino más bien de ejecución de las resoluciones en materia de responsabilidad parental, saltan a la vista algunos de los principales problemas y retos del proceso de integración europea en este ámbito.

\subsection{El régimen general de reconocimiento y ejecución de las resoluciones en materia de responsabilidad parental del Reglamento Bruselas II bis}

En general, todas las resoluciones en materia de responsabilidad parental dentro del ámbito de aplicación del Reglamento Bruselas II bis gozan de un reconocimiento automático en los Estados miembros de la UE. Es decir, las decisiones en materia de responsabilidad parental poseen los mismos efectos procesales que el Estado de origen les atribuye en todo el territorio de la UE, desde el momento en que se dicta dicha resolución y sin necesidad de procedimiento alguno.

Concretamente, una vez dictada una resolución en un Estado miembro, las partes interesadas pueden solicitar, en otro Estado miembro, que se resuelva sobre el reconocimiento o no reconocimiento de dicha resolución. El artículo 23 del Reglamento detalla las condiciones específicas para el reconocimiento de la decisión sobre responsabilidad parental. Además, en este precepto se recoge un listado de motivos de denegación del reconocimiento.

En cuanto a la ejecución, para poder ejecutar las resoluciones en materia de responsabilidad parental se deberá solicitar la declaración de ejecutividad o exequátur. Así, para dotar a una resolución extranjera de 
fuerza ejecutiva y convertirla en título ejecutivo, se debe instar un procedimiento específico, unilateral y ex parte. Por tanto, todas estas decisiones dictadas en un Estado miembro deberán necesariamente someterse a unas exigencias formales para beneficiarse del sistema de eficacia extraterritorial previsto en el Reglamento Bruselas II bis.

\subsection{Régimen especial de ejecución en materia de sustracción internacional de menores y derechos de visita}

Como anticipábamos supra, el Reglamento Bruselas II bis ha introducido una novedosa modalidad específica de eliminación del exequátur que solo será aplicable en supuestos de derechos de visita (artículo 41) y restitución del menor (artículo 42). Esta modalidad habilita la ejecutividad inmediata de las decisiones de otro Estado miembro en los supuestos mencionados. Es decir, al eliminar el procedimiento de exequátur, la resolución en cuestión poseerá fuerza ejecutiva directa, sin necesidad de este procedimiento. A ello se le añade la imposibilidad de impugnación de tal resolución, siempre que haya sido certificada en el Estado miembro de origen, como detallaremos posteriormente.

Es precisamente esta segunda modalidad la que afecta a la sustracción internacional de menores. Supone una gran innovación respecto al sistema previsto por el Convenio de La Haya de 1980, ya que, como se indicó supra, este no contempla un régimen de reconocimiento y ejecución de las resoluciones judiciales que deciden sobre el retorno del menor.

\subsubsection{El mecanismo de retorno o de última palabra del artículo 11.8}

A continuación, nos centraremos en las órdenes de retorno del menor tras un traslado o retención ilícita, dictadas en virtud del mecanismo de prevalencia. La eliminación del exequátur para este tipo de resoluciones ha tenido gran repercusión en la práctica, razón por la cual es esencial comprender las consecuencias de este novedoso sistema de ejecución.

Sin embargo, antes de entrar a analizar dichas consecuencias, se debe tener perfectamente delimitado el supuesto de hecho que puede llevar a la emisión de una resolución de retorno ejecutiva sin necesidad de exequátur, así como los requisitos para la emisión del certificado pertinente.

En primer lugar, el artículo 42 del Reglamento Bruselas II bis prevé la eliminación del exequátur para la resolución de restitución emitida de conformidad con el artículo 11.8 del Reglamento. Así, la eliminación del exequátur afecta exclusivamente a la resolución de restitución de un menor, dictada por el Estado miembro competente -que normalmente será el Estado miembro de residencia habitual inmediatamente anterior al traslado o retención ilícita-, con posterioridad a una resolución de no restitución del menor, emitida por el Estado requerido -Estado miembro en el que el menor se encuentra ilícitamente-, resolución que se ha basado en un motivo de excepción del Convenio de La Haya de 1980.

Incidiendo en este punto, es muy importante entender que el artículo 42 únicamente será de aplicación ante una previa resolución de no restitución dictada en un Estado miembro, que normalmente será aquel en el que se encuentra el menor que ha sido trasladado o retenido de forma ilícita. Es decir, solo en el caso de que no se consiga obtener la restitución del menor mediante la acción de restitución directa del menor -ante las autoridades del Estado miembro donde se encuentra el menor que ha sido presuntamente trasladado de modo ilícito-, podremos acudir de forma subsidiaria a este mecanismo (Calvo y Carrascosa, 2018, p. 629 y ss). 
En cuanto a las exigencias para obtener dicha resolución de restitución con eficacia ejecutiva directa, se precisa la expedición de una certificación. La certificación se expedirá de oficio por el juez de origen, cumplimentando el formulario del anexo IV del Reglamento. Además, requiere que se cumplan las siguientes condiciones: que se haya dado al menor la posibilidad de audiencia, teniendo en cuenta su edad y madurez; que se haya dado posibilidad de audiencia a las partes y que el juez haya tenido en cuenta, en su sentencia, las razones y pruebas en las que se fundamenta resolución de no restitución del menor, emitida en virtud del artículo 13 del Convenio de La Haya de 1980.

El carácter directamente ejecutivo de la resolución de restitución del menor tiene por consecuencia principal la limitación al máximo del margen de actuación del órgano jurisdiccional del Estado miembro requerido. Así, el Reglamento refuerza hasta tal punto el sistema de certificación que:

1) No existe ningún motivo de denegación de la ejecución que faculte a los órganos del Estado miembro requerido para rechazar la ejecución de la resolución de retorno del menor. Por tanto, no puede ejercer ningún tipo de control sobre las condiciones de emisión del certificado que expide el Estado de origen.

2) La decisión que acuerda la ejecución de la resolución de retorno del menor, tomada por el órgano del Estado miembro donde se encuentra el menor, no puede ser objeto de recurso alguno.

3) Frente a la resolución de retorno del menor, certificada conforme al artículo 42.2 del Reglamento, solo cabe instar un procedimiento de rectificación o dudas en cuanto a su autenticidad, que debe llevarse a cabo en el Estado de origen (artículo 43 del Reglamento).

De estos puntos se extrae que, una vez emitido el certificado, ya no es posible impugnar el reconocimiento en el Estado requerido, ni tampoco su expedición. Así, el juez del Estado requerido solo deberá verificar que dicho certificado reúne dichas condiciones para aceptarlo, siendo el único requisito para que la resolución sea reconocida y posea fuerza ejecutiva en dicho Estado.

\subsubsection{Deficiencias del mecanismo de retorno: la garantía del interés superior del menor en el caso concreto}

De esta manera, lo que caracteriza este nuevo modelo es que ya no es el juez del Estado miembro requerido el que procede al control de la ejecutividad de la resolución de retorno del menor, sino que es el propio juez del Estado miembro de origen el que realiza dicho control, mediante la expedición de un certificado que deberá cumplir las condiciones señaladas. Vemos, por tanto, cómo se ha alterado el método tradicional, ya que el control de la resolución extranjera se realiza a priori en el Estado de origen y no a posteriori en el Estado requerido.

En este sentido, muchos autores se han mostrado críticos con la eliminación del exequátur en términos absolutos, sin siquiera la posibilidad de alegar contrariedad con el orden público. Fundamentalmente, se entiende como una medida prematura para la cual el espacio judicial europeo no está preparado, dada la falta de armonización en las normas procesales y materiales, y la consecuente divergencia entre las legislaciones nacionales de los distintos Estados miembros (Espinosa, 2007, p. 223).

Concretamente, algunos autores resaltan lo confuso que es pensar que dos jueces de Estados miembros diversos ordenen decisiones diversas, basándose ambos en el Reglamento Bruselas II bis, que a su vez remite al Convenio de La Haya de 1980 (Espinosa, 2007, p. 223). La justificación dada es que la resolución posterior de regreso del menor es ejecutiva con el fin de garantizar la restitución del menor. Sin embargo, 
esta competencia exclusiva del juez de origen, sin siquiera un motivo de oposición de la ejecución basado en el orden público o en el interés superior del menor en el caso concreto, puede desencadenar ciertos problemas en torno a la protección de los derechos fundamentales del menor, así como a la consideración de su interés superior en un caso concreto (Espinosa, 2016, p. 352).

Estos problemas interpretativos se han evidenciado particularmente a partir de la jurisprudencia TJUE y el TEDH ${ }^{28}$. El TJUE ha respaldado la aplicación del artículo 11.8 del Reglamento Bruselas II bis, sin excepciones $^{29}$. Por el contrario, el TEDH en algunos pronunciamientos ${ }^{30}$ en la materia pareció reivindicar una evaluación en concreto del interés superior del menor, más allá de su mera restitución (Bouza, 2012, p. 213). En definitiva, ha surgido el debate en torno a si en los supuestos de secuestro internacional de menores no debería involucrarse en un mayor análisis del fondo de la cuestión debatida, analizando cuál es el interés superior del menor en cada caso, más allá de una simple restitución automática (Cuniberti, 2014, p. 303 y ss; Forcada, 2016a, p. 32).

\section{Reglamento 2019/1111: ampliación de la eliminación del exequátur a todas resoluciones en materia de responsabilidad parental}

\subsection{Introducción}

Tras más de diez años de aplicación del Reglamento Bruselas II bis, en el año 2016 se inició un procedimiento de revisión del texto reglamentario mediante la presentación, el 30 de junio de 2016, de la Propuesta de Refundición del Reglamento Bruselas II bis por la Comisión Europea. El objetivo principal de la propuesta se sitúa en dos ejes fundamentales: por un lado, el fortalecimiento del principio de reconocimiento mutuo y de la libre circulación de resoluciones judiciales; y, por otro, la mejor protección del interés superior del menor $^{31}$. Este proceso de reforma culminó en junio de 2019 con la aprobación del texto definitivo del nuevo Reglamento Bruselas II ter ${ }^{32}$.

Del estudio realizado por la Comisión se extrae que, de los dos principales ámbitos cubiertos por el Reglamento Bruselas II bis, cuestiones matrimoniales y cuestiones de responsabilidad parental, estas últimas son las que han planteado graves problemas en la práctica. En relación con el objeto del presente trabajo, se detectan deficiencias en las siguientes materias: el procedimiento de restitución de menores, el requisito del exequátur y la ejecución efectiva de las decisiones.

28 Para un estudio de esta problemática véase González Marimón (2019a).

29. Véase STJUE de 11 de julio de 2008, C-A195/08, Igna Rinau, STJUE de 1 de julio de 2010, C-211/201, Povse c./ Alpago, STJUE de 22 de diciembre de 2010, C-491/PPU/2010, Aguirre Zárraga.

30 Véase STEDH de 6 de julio de 2010, demanda n. ${ }^{\circ}$ 41615/07, Neulinger y Shuruk contra Suiza; STEDH de 9 de septiembre de 2010, demanda n. ${ }^{\circ}$ 25437/08, Raban contra Rumania; STEDH de 15 de mayo de 2012, M.R y M.L contra Estonia; STEDH de 26 de noviembre de 2013, demanda n. ${ }^{\circ} 27853 / 09$, X contra Letonia.

31 En palabras de la Propuesta: "seguir desarrollando el espacio europeo de justicia y derechos fundamentales basado en la confianza mutua, suprimiendo los obstáculos que subsisten a la libre circulación de las resoluciones, en consonancia con el principio de reconocimiento mutuo, así como proteger mejor el interés superior del nińo mediante la simplificación de los procedimientos y la mejora de su eficacia" (Propuesta de Refundición del Reglamento Bruselas II bis, p. 2).

32 Sobre las novedades introducidas en el Reglamento Bruselas II ter, véase Borrás Rodríguez (2019), Borrás (2020), Forcada (2020), Rodríguez Pineau (2020), González Beilfuss (2020), Carpaneto (2019), Biagioni (2019), Campuzano (2019), Pardo (2020) y Puig (2020). 
Respecto a las principales áreas finalmente reformadas, cabe destacar las siguientes:

En primer lugar, se mantiene el sistema de retorno del menor en caso de un traslado o retención ilícito de un menor. Este decir, se mantiene la remisión al Convenio de La Haya de 1980 y, a su vez, se mantiene el mecanismo de prevalencia o de última palabra. Ahora bien, en ambos casos se introducen una serie de medidas para mejorar su funcionamiento en la práctica y evitar algunos de los problemas detectados a partir de la práctica jurisprudencial. Para ello, el nuevo Reglamento contiene un nuevo capítulo (capítulo III) dedicado exclusivamente a la sustracción internacional de menores.

En segundo lugar, la mayor novedad de la Refundición del Reglamento Bruselas II bis es el avance en el objetivo final de la libre circulación de resoluciones judiciales entre los Estados miembros, mediante la eliminación de la declaración de ejecutividad para todas las resoluciones en materia de responsabilidad parental. La Comisión Europea entiende que la cooperación judicial y la confianza entre los Estados miembros han alcanzado un grado de madurez que permite dar el paso hacia un sistema más simple en la circulación de resoluciones. Siguiendo el modelo de otros reglamentos europeos -como por ejemplo el Reglamento Bruselas I bis33- el legislador europeo decide extender la supresión del exequátur a todas las decisiones en materia de responsabilidad parental, pero salvaguardando los derechos procesales de las partes. Por tanto, como a continuación se desarrollará, las resoluciones de retorno del menor pasan a estar incluidas en este régimen general, pero con algunas especificidades.

\subsection{Régimen general: eliminación del exequátur de todas las resoluciones en materia de responsabilidad parental}

El capítulo IV del Reglamento Bruselas II ter recoge el régimen de reconocimiento y ejecución de las resoluciones extranjeras, distinguiendo entre un régimen general de reconocimiento y ejecución (sección 1) y un régimen privilegiado de reconocimiento y ejecución para ciertas resoluciones en materia de responsabilidad, que seguirán siendo las relativas a los derechos de visita y de restitución del menor en caso de sustracción internacional de menores (sección 2). A estos dos regímenes le acompañan unas disposiciones comunes sobre la ejecución (sección 3).

El reconocimiento de resoluciones continúa siendo prácticamente igual a la regulación vigente: reconocimiento automático y reconocimiento incidental. Cualquiera de las partes podrá solicitar el reconocimiento o el no reconocimiento, y como novedad se introduce la posibilidad de solicitar que se resuelva que no hay motivos para la denegación del reconocimiento (artículo 30.3). En el artículo 39 se recogen las causas tasadas de oposición al reconocimiento en materia de responsabilidad parental.

La supresión del exequátur para todas las resoluciones en materia de responsabilidad parental se recoge en el nuevo artículo 34 (apartado 1), estableciendo que las resoluciones que sean ejecutivas en un Estado miembro lo serán también en otro, sin necesidad de una declaración de fuerza ejecutiva. Así, se elimina el procedimiento formal, pero no se llega a una supresión total del exequátur, como ocurre con las actuales órdenes de retorno del artículo 11.8 del Reglamento Bruselas II bis. Por el contrario, el legislador de la UE ha seguido la línea de otros reglamentos europeos y ha mantenido la oposición de la ejecución; es decir, por una supresión del exequátur con salvaguardias (Rodríguez Vázquez, 2017, p. 779).

33 Reglamento (UE) N ${ }^{\circ}$ 1215/2012 del Parlamento Europeo y del Consejo, de 12 de diciembre de 2012, relativo a la competencia judicial, el reconocimiento y la ejecución de resoluciones judiciales en materia civil y mercantil, DO L 351/1, de 20 de diciembre de 2012. 
El Reglamento Bruselas II ter, paralelamente a la eliminación del exequátur, refuerza y extiende el sistema de certificados para todas las resoluciones en materia de responsabilidad parental (artículo 36.1 b), debiendo rellenarse el formulario del Anexo III del nuevo Reglamento.

\subsection{Especialidades en materia de sustracción ilícita internacional}

A pesar de la ampliación de la eliminación del exequátur a todas las resoluciones en materia de responsabilidad parental, como se indica supra, el legislador de la UE ha querido seguir dotando de un régimen privilegiado a las resoluciones que ordenen la restitución del menor, siguiendo el mecanismo de prevalencia. Efectivamente, en la sección 3 del capítulo IV se detalla el régimen especial de tales resoluciones en el reconocimiento y la ejecución. Esencialmente, este régimen privilegiado contiene las siguientes especialidades:

El reconocimiento no solo será automático, sino que además lo será sin que quepa alegar ningún motivo de oposición al reconocimiento, salvo el caso de resoluciones irreconciliables que prevé el artículo 50.

Igualmente, la ejecución será automática, sin necesidad de declaración de ejecutividad y con la única posibilidad de oposición a la ejecución el caso de las resoluciones irreconciliables del artículo 50.

Además, se mantiene un certificado especial para el régimen privilegiado (certificado del artículo 47 en relación con el anexo VI). En relación con este punto, se exige un requisito adicional para la emisión del certificado relativo a las resoluciones de retorno del menor, derivadas del mecanismo de prevalencia. El juez deberá justificar que ha tenido en cuenta las razones y pruebas en que se basa la resolución anterior dictada en virtud del artículo 13 del Convenio de La Haya de 1980 por el Estado miembro en el que se encuentra ilícitamente el menor.

Más allá de la restricción de los motivos tanto del reconocimiento como de la ejecución, la gran singularidad en materia de sustracción internacional de menores la encontramos en la sección 3 del capítulo IV, titulada "Disposiciones comunes sobre ejecución”. Dentro de esta sección, el legislador de la UE introduce la posibilidad de suspender el procedimiento de ejecución en el artículo 56. La gran revolución la encontramos en su apartado $4^{\circ}$, el cual permite, en casos excepcionales, que se suspenda el procedimiento de ejecución en caso de que esta "exponga al menor a un riesgo grave de daño físico o psíquico debido a impedimentos temporales que hayan surgido después de que la resolución haya sido dictada, o en virtud de cualquier otro cambio significativo de circunstancias". La ejecución deberá reanudarse en cuanto deje de existir tal riesgo para el menor y solo cuando tal riesgo tenga "carácter duradero" se podrá denegar la ejecución.

El legislador europeo está revocando la eliminación absoluta del exequátur que había diseñado para el mecanismo de prevalencia del artículo 11.8 del Reglamento Bruselas II bis. El Estado miembro requerido contará con un cierto margen de maniobra, reestableciendo un cierto equilibrio en el reparto de competencias entre Estado de origen y Estado requerido. Con ello, el legislador de la UE responde a las críticas derivadas de la interpretación formalista del TJUE y opta por prever una solución para supuestos excepcionales en los que el interés superior del menor en el caso concreto recomienda la no restitución del menor, siguiendo el criterio del TEDH.

Se ha demostrado que un sistema de ejecución automática sin posibilidad alguna de oposición a la ejecución no garantiza suficientemente la protección de los derechos fundamentales del menor y menos sin una mínima armonización entre Estados miembros. Por ello, se ha introducido la posibilidad, en última 
instancia, de suspender la ejecución como segundo control del interés superior del menor, reconociendo la influencia del factor tiempo en la conformación de este interés.

Solo falta por ver cuál será la realidad práctica en la interpretación y aplicación de esta nueva posibilidad de suspensión de la ejecución como medida excepcional de protección del interés superior del niño. Pues, precisamente en este punto, radica el problema de fondo: la interpretación del principio del interés superior del menor. Al ser un concepto jurídico indeterminado que debe determinarse atendiendo a las circunstancias del caso concreto, puede llevar a justificar tanto el retorno como el no retorno del menor, dependiendo de tales circunstancias.

Pese a todo lo dicho, lo cierto es que el sistema no estaba funcionando, tal y como se ha evidenciado tanto por la jurisprudencia del TJUE como del TEDH. Ante esta situación, el legislador de la UE ha dado una respuesta que palía los problemas surgidos en la práctica. A través del ejemplo de la sustracción internacional de menores se puede apreciar cómo el legislador europeo avanza en la integración europea de los procesos de familia, mediante avances y retrocesos, mediante la técnica ensayo-error. Se quiso correr demasiado a través de la eliminación absoluta del exequátur, sin ningún motivo de oposición. El legislador europeo ha dado por tanto un paso atrás, pero sin renunciar al gran avance que supone la eliminación del exequátur para todas las resoluciones en materia de responsabilidad parental.

En definitiva, desde la perspectiva de la protección de los derechos fundamentales, se puede afirmar que el nuevo Reglamento es más garantista con tales derechos en general, y, en concreto, con los derechos del menor. Con ello, el texto Reglamentario se acerca a los estándares tanto del TEDH como del Comité de Derechos del Niño. Y en esta línea, la doctrina está de acuerdo en afirmar que la eliminación del exequátur con garantías procesales y, en particular, para la protección del interés superior del menor, mejoran el sistema anterior (Beaumont et al., 2016, p. 10; Gandía, 2017, pp. 805-806; GEDIP, 2016, Rodríguez Pineau, 2017, p. 152; Rodríguez Vázquez, 2017, p. 782).

\section{Conclusión}

La consecución del objetivo final de la libre circulación de resoluciones judiciales en la UE constituye, aún en la actualidad, un gran reto para el legislador de la UE. Este objetivo, materializado en el reconocimiento y la ejecución automática -sin necesidad de un procedimiento de exequátur o declaración de ejecutividadse asienta en principios esenciales de la Unión, como el principio de reconocimiento mutuo y la confianza judicial. Ahora bien, este loable objetivo sigue enfrentándose a grandes retos, comunes en el proceso de integración europea, como es la ausencia de una armonización mínima de normas procesales y materiales.

Dentro de esta gran tendencia, la materia de la sustracción internacional de menores es un ejemplo paradigmático de la paulatina evolución de la libre circulación de resoluciones judiciales en la UE. El legislador de la UE tomó la decisión de hacer frente a este fenómeno en normas institucionales. Sin embargo, no pudo dejar de tomar en consideración los instrumentos convencionales ya existentes en la materia. Y, por tanto, tuvo que diseñar un régimen que no solo avanzara en la libre circulación de resoluciones judiciales en la UE y garantizara el interés superior del menor, sino que además estuviera en sintonía con el sistema ya diseńado por el Convenio de La Haya de 1980.

Este difícil equilibrio se intentó alcanzar en el Reglamento Bruselas II bis mediante el refuerzo de las competencias del Estado de origen, normalmente el de residencia habitual del niño. Y, en concreto, mediante la eliminación del exequátur de las resoluciones de retorno dictadas en virtud del artículo 11.8 
del Reglamento, sin posibilidad de oposición a la ejecución. Pese a ser un gran avance desde el punto de vista de la integración europea, en la práctica pareció no garantizar el interés superior del menor en todos los casos.

Este es uno de los motivos por los que, finalmente, en el nuevo Reglamento Bruselas II ter, a pesar de continuar con el objetivo de la libre circulación de resoluciones judiciales, no se ha seguido el modelo anterior. Por el contrario, se han mantenido ciertos motivos de oposición al reconocimiento y a la ejecución en materia de responsabilidad parental. Y, aunque en la materia específica de la sustracción internacional de menores se mantiene el mecanismo de prevalencia, se abre una vía de escape en sede de ejecución. La posibilidad, en circunstancias excepcionales, de suspender y, en última instancia, denegar la ejecución en caso de que entrañe un grave riesgo del menor debido a un cambio de circunstancias.

En definitiva, a la espera de que el nuevo Reglamento entre en aplicación y podamos comprobar su aplicación e interpretación en la práctica, podemos concluir que el legislador de la UE ha conseguido seguir avanzando en la integración europea, pero encontrando un mejor equilibrio entre la libre circulación de resoluciones judiciales y el interés superior del menor.

\section{Bibliografía citada}

Ancel, Bertrand y Muir Watt, Horatia (2005): "L'intérêt supérieur de l'enfant dans le concert des jurisdictions: le Reglèment Bruxelles II bis”, Revue Critique de Droit International Privé, pp. 569605.

Beaumont, Paul, Walker, Lara y Holliday, Jayne (2016): "Parental Responsibility and International Child Abduction in the proposed recast of Brussels IIa Regulation and the effect of Brexit on future child abduction proceedings", University of Aberdeen, Working Paper 6. Disponible en https://www.abdn. ac.uk/law/documents/CPIL_Working_Paper_No_2016_6_revised.pdf [Fecha de consulta: 18 de noviembre de 2020].

Bouza Vidal, Nuria (2012): “La integración del Convenio de La Haya de 1980 sobre sustracción internacional de menores en el sistema europeo de Derechos Humanos", en Esplugues Mota, Carlos y Palao Moreno, Guillermo (coordinadores), Nuevas fronteras del Derecho de la Unión Europea. Liber amicorum José Luis Iglesias Buhigues (Valencia, Tirant Lo Blanch), pp. 203-218.

Calvo Caravaca, Alfonso y Carrascosa González, Javier (2018): Derecho Internacional Privado (Granada, Comares, $\left.18^{\mathrm{a}} \mathrm{ed}.\right)$, Volumen II.

Caro Gándara, Rocío (2010): "Desconfianza comunitaria a la luz de la jurisprudencia del Tribunal de Justicia sobre el Reglamento Bruselas II bis: algunas claves para el debate", Anuario Español de Derecho Internacional Privado, X: pp. 417-439.

Cuniberti, Gilles (2014): “Abolition de l'exequatur et présomption de protection des droits fondamentaux", Revue Critique de Droit International Privé, 103, 2: pp. 303-327.

Espinosa Calabuig, Rosario (2007): Custodia y visita de menores en el espacio judicial europeo (Madrid, Marcial Pons).

Espinosa Calabuig, Rosario (2016): “Traslado o retención ilícitos de menores tras la reforma de 2015: rapidez, especialización y... algunas ausencias”, Revista Española de Derecho Internacional, 68, 2: pp. 347-357.

Esplugues Mota, Carlos, Iglesias Buhigues, José Luis y Palao Moreno, Guillermo (2020): Derecho Internacional Privado (Valencia, Tirant Lo Blanch, 14a ed.). 
Forcada Miranda, Francisco-Javier. (2016 a): "El nuevo proceso español de restitución o retorno de menores en los supuestos de sustracción internacional: La decidida apuesta por la celeridad y la novedosa Circular de la Fiscalía 6/2015 (Partes I y 2)”, Bitácora Millennium DIPr. Disponible en www.milleniumdipr.com [Fecha de consulta: 18 de noviembre de 2020].Gandía Sellens, María Aránzazu (2017): "La responsabilidad parental y la sustracción de menores en la propuesta de la Comisión para modificar el RB II bis: algunos avances, retrocesos y ausencias”, Anuario Español de Derecho Internacional Privado, XVII: pp. 799-820.

GEDIP (Subgrupo Nacionalidad) (2016): “Comments on the Commission Proposal for a Council Regulation on jurisdiction, the recognition and enforcement of decisions in matrimonial matters and the matters of parental responsibility, and on international child abduction (recast) (COM (2016) 411 final)". Disponible en https://www.gedip-egpil.eu/reunionstravail/Reunion\%2026/ Annexe\%20I.pdf [Fecha de consulta: 18 de noviembre de 2020].

Iglesias Buhigues, José Luis y Desantes Real, Manuel (1986): “La quinta libertad comunitaria: competencia judicial, reconocimiento y ejecución de resoluciones judiciales en la Comunidad Europea”, en García De Enterría, Eduardo, González Campos, Julio y Muñoz Machado, Santiago (editores), Tratado de Derecho Comunitario Europeo (Madrid, Civitas, Tomo III), pp. 711-752.

López De Tejada Ruiz, María (2011): “La supresión del exequátur en el espacio judicial europeo”, Diario La Ley. Sección Tribuna, 776.

Magnus, Ulrich Y Mankowski, Peter (2012): Brussels IIbis Regulation (Munich, Sellier European Law Publishers).

Mceleavy, Peter (2005): "The new Child Abduction Regime in the European Union: Symbolic Relationship or Forced Partnership?", Journal of Private International Law, 1: pp. 5-34.

Reig Fabado, Isabel (2008): “Incidencia del Reglamento 2201/2003 en materia de sustracción internacional de menores: interacción con el Convenio de La Haya de 1980”, en Llória García, Paz (director), Secuestro de menores en el ámbito familiar: un estudio interdisciplinar (Madrid, Iustel), pp. 219-242.

Rodríguez Pineau, Elena (2017): "La refundición del Reglamento Bruselas II bis: de nuevo sobre la función del Derecho Internacional privado europeo", Revista Española de Derecho Internacional, Sección Estudios, 69, 1: pp.139-165.

Rodríguez Vázquez, María Ángeles (2017): "La reforma del Reglamento (CE) n.o 2201/2003: análisis de las soluciones propuestas en materia de reconocimiento y ejecución de resoluciones", Anuario Español de Derecho Internacional Privado, XVII: pp. 767-784.

Bibliografía sugerida

antiago

Arenas García, Rafael (2019): “Cinco décadas de proceso codificador en la UE: historia de un éxito”, en Esplugues Mota, Carlos., Diago Diago, Pilar y Jiménez Blanco, Pilar (editores), 50 años de Derecho Internacional Privado de la Unión Europea en el diván (Valencia, Tirant Lo Blanch), pp. 23-51.

Beaumont, Paul y Mceleavy, Peter (1999): The Hague Convention on International Child Abduction, (Oxford, Oxford University Press).

Biagioni, Giacomo (2019): “Il nuovo regolamento (UE) 2019/1111 relativo alla competenza, al ricognoscimento e all'esecuzione delle decisioni in materia matrimoniale e di responsabilità genitoriale, e alla sottrazione internazionale”, Rivista di diritto internazionale, 102, 4: pp. 11691178.

Borrás Rodríguez, Alegría (2019): “Bruselas II, Bruselas II bis, Bruselas II ter...”, Revista Electrónica de Estudios Internacionales, 38: pp. 1-5. 
Borrás Rodríguez, Alegría (2020): "La sustracción internacional de menores: del Convenio de La Haya de 1980 al Reglamento Bruselas II ter”, en AA.VV., El Derecho Internacional privado entre la tradición y la innovación. Libro homenaje al Profesor Doctor José María Espinar Vicente (Madrid, Iprolex), pp. 159-174.

Caamiña Domínguez, Cecilia (2011): “La supresión del exequátur en el R. 2201/2003”, Cuadernos de Derecho Transnacional, 1: pp. 63-83.

Campuzano Díaz, Beatriz (2013): "La política legislativa de la UE en DIPR de Familia. Una visión de conjunto", Cuadernos de Derecho Transnacional, 5, 2: pp. 234-264.

Campuzano Díaz, Beatriz (2019): "El nuevo Reglamento (UE) 2019/1111: análisis de las mejoras en las relaciones con el Convenio de La Haya de 19 de octubre de 1996 sobre responsabilidad parental", Cuadernos de Derecho Transnacional, 12, 1: pp. 97-117.

Caro Gándara, Rocío (2011): "De la desconfianza recíproca al reconocimiento mutuo: una laboriosa transición (El Reglamento Bruselas II bis como banco de pruebas)”, Diario La Ley. Sección Doctrina, 8395.

Carpaneto, Laura (2019): "Impact of the Best Interests of the Child on the Brussels II ter Regulation", en Bergamini, Elisabetta Y Ragni, Chiara (editores): Fundamental Rights and Best Interests of the Child in Transnational Families, (Cambridge - Antwerp - Chicago, Intersentia), pp. 265-286.

Comisión Europea: Guia práctica para la aplicación del nuevo "Reglamento Bruselas II bis". Disponible en http://publications.europa.eu/resource/cellar/f7d39509-3f10-4ae2-b993-53ac6b9f93ed.0007.01/ DOC_1 [Fecha de consulta: 18 de noviembre de 2020].

De Hoyos Sancho, Montserrat (2007): "El principio de reconocimiento mutuo como principio rector de la cooperación judicial europea”, en Jimeno Bulnes, M, La cooperación judicial civil y penal en el ámbito de la Unión Europea: instrumentos procesales (Universidad de Burgos, Editorial Bosch) pp. 67-93.

Font Segura, Albert (2004): "El progresivo avance del derecho comunitario en materia de familia: un viaje inconcluso de Bruselas II a Bruselas II bis", Revista Española de Derecho Internacional: 56, 1: 273-299.

Forcada Miranda, Francisco-Javier. (2016 b): “Complejidad, carencias y necesidades de la sustracción internacional de menores en el siglo XXI y un nuevo marco legal en España”, Anuario Español de Derecho Internacional Privado, XVI: pp. 699-743.

Forcada Miranda, Francisco-Javier. (2020.): Comentarios prácticos al Reglamento (UE) 2019/1111. Competencia, Reconocimiento y Ejecución de Resoluciones en materia Matrimonial, Responsabilidad Parental y Sustracción Internacional de Menores (Madrid, Sepín).

Garau Sobrino, Federico (2004): "La declaración de ejecutividad automática. ¿Hacia una nueva teoría general del exequátur?”, Anuario Español de Derecho Internacional Privado: pp. 91-116.

Gayo, Mercedes (2013): "El reconocimiento automático de sentencias ya es una realidad en la UE", Actualidad Jurídica Aranzadi, 858: pp. 8 y ss.

Gómez Jene, Miguel (2006): "La cooperación judicial en materia civil”, Revista de Derecho de la Unión Europea, 10: pp. 139-154.

González Beilfuss, Cristina (2004): "Relaciones e interacciones entre Derecho Comunitario, Derecho Internacional Privado y Derecho de familia europeo en la construcción de un espacio judicial común", Anuario Español de Derecho Internacional Privado, IV: pp. 117-186.

González Beilfuss, Cristina (2020): “La sustracción de menores en el nuevo Reglamento 2019/1111”, en AA.VV., Relaciones transfronterizas, globalización y Derecho. Homenaje al Prof. Dr. José Carlos Fernández Rozas, (Cizur Menor, Navarra, Civitas-Thomson Reuters), pp. 383- 398. 
González Marimón, María (2019 a): “El 'diálogo’ entre el TJUE y el TEDH en torno a la eliminación del exequátur del mecanismo de retorno del Reglamento Bruselas II bis”, en AAVV, El mercado único en la Unión Europea. Balance y perspectivas jurídico-politicas (Madrid, Dykinson), pp. 81-94.

González Marimón, María (2019 b): "El principio del interés superior del menor en supuestos de sustracción ilícita internacional: la jurisprudencia del TJUE y del TEDH”, en García Garnica, María del Carmen y Marchal Escalona, Nuria (directores), Aproximación interdisciplinar a los retos actuales de protección de la infancia dentro y fuera de la familia (Madrid, Aranzadi), pp. 637- 658.

Guzmán Peces, Montserrat (2014-2015): "Problemática en la coordinación de los instrumentos normativos aplicable a la sustracción de menores y en particular a la interpretación de la residencia habitual”, Anuario Español de Derecho Internacional Privado, XIV-XV: pp. 489-522.

Herranz Ballesteros, Mónica (2012): “Los Tribunales de Estrasburgo y Luxemburgo ante la protección de los derechos fundamentales en supuestos de sustracción internacional de menores", Revista de Derecho Europeo: pp. 41-60.

Hess, Burkhard (2014-2015): "La influencia del Tribual Europeo de Derechos Humanos en el Derecho Procesal Civil europeo", Anuario Español de Derecho Internacional Privado, XIV-XV: pp. 35-53.

Iglesias Buhigues, José Luis (1998): "La cooperación judicial en materia civil (CJC) antes y después del Tratado de Ámsterdam”, Revista General de Derecho, 644: pp. 5847-5862.

Iglesias Buhigues, José Luis (2010): "Reflexiones en torno al espacio europeo de justicia. A propósito del futuro reglamento en materia sucesoria”, Revista valenciana d'estudis autonòmics, 54, 2: pp. 276295.

Lapiedra Alcamí, Rosa (2008): "La sustracción internacional de menores: el Convenio de La Haya de 25 de octubre de 1980", en Llória García, Paz (director), Secuestro de menores en el ámbito familiar: un estudio interdisciplinar (Madrid, Iustel), pp. 189-218.

López Guerra, Luis (2017): "El Tribunal Europeo de Derechos Humanos, el Tribunal de Justicia de la UE y «Le mouvement nécessaire des choses»", UNED, Teoria y Realidad Constitucional, 39: pp. 163-188.

Michinel Álvarez, Miguel Ángel (2013): “La cooperación judicial en materia civil en el Espacio de Libertad, Seguridad y Justicia”, en Sánchez Ramos, Belén (coordinador), La Unión Europea como actor global: algunas cuestiones analizadas desde el Tratado de Lisboa (Valencia, Tirant Lo Blanch), pp. 247-274.

Ordónez Solís, David (2014): "La configuración del Espacio Judicial Europeo”, Cuadernos Europeos de Deusto, 50: pp. 105-139.

Pardo Iranzo, Virginia (2020): "Responsabilidad parental y sustracción internacional de menores: la ejecución en el nuevo Reglamento 2019/1111”, Diario LA LEY, 9629: pp. 1-14.

Pérez Vera, Elisa (1982): “Informe Explicativo”. Disponible en https://www.hcch.net/es/publicationsand-studies/details4/?pid=2779 [Fecha de consulta: 18 de noviembre de 2020].

Pertegás Sender, Marta (2019): “El proceso de codificación del DIPr en la Unión Europea y su interacción con otros procesos de codificación: La Haya”, en Esplugues Mota, Carlos., Diago Diago, Pilar y Jiménez Blanco, Pilar (editores), 50 años de Derecho Internacional Privado de la Unión Europea en el diván (Valencia, Tirant Lo Blanch), pp. 175-194.

Porcheron, Delphine (2015): "La jurisprudence des deux Cours européennes (CEDH et CJUE) sur le déplacement illicite d'enfant: vers une relation de complémentarité?", Journal du Droit International Lexinexis, 142: pp. 821-844.

Puig Blanes, Francisco de Paula (2020): "Aproximación al tratamiento de la sustracción internacional de menores en el Reglamento UE 2019/1111”, Actualidad Civil, 4: pp. 1-9. 
Reig Fabado, Isabel (2018): "El traslado ilícito de menores en la Unión Europea: retorno vs. violencia familiar o doméstica", Cuadernos de Derecho Transnacional, 10, 1: pp. 610-619.

Rodríguez Pineau, Elena (2020): “El nuevo Reglamento (UE) 2019/1111 en materia matrimonial, responsabilidad parental y sustracción internacional de menores", LA LEY Derecho de familia, 26: pp. 1-26.

Sánchez Lorenzo, Sixto (2012): “La política legislativa de la Unión Europea en materia de Derecho internacional privado: de la técnica del carro ante los bueyes a la estrategia del avestruz", en Esplugues Mota, Carlos y Palao Moreno, Guillermo (coordinadores), Nuevas fronteras del Derecho de la Unión Europea, Liber amicorum José Luis Iglesias Buhigues (Valencia, Tirant Lo Blanch) pp. 133-146.

Sánchez Lorenzo, Sixto (2019): "Cincuenta años de Derecho Internacional Privado de la Unión Europea: el lado oscuro", en Esplugues Mota, Carlos., Diago Diago, Pilar y Jiménez Blanco, Pilar (editores), 50 años de Derecho Internacional Privado de la Unión Europea en el diván (Valencia, Tirant Lo Blanch), pp. 53-86.

Trimmings, Katarina (2013): Child Abduction within the European Union (Oxford and Portland, Hart Publishing). 